CUBO A Mathematical Journal

Vol.16, Noㅡ, (37-53). October 2014

\title{
Existence Results for Some Neutral Partial Functional Differential Equations of Fractional order with State-Dependent Delay
}

\author{
MOUFFAK BenCHOHRA \\ Laboratory of Mathematics, \\ University of Sidi Bel Abbes, \\ PO Box 89, 22000 Sidi Bel \\ Abbes, Algeria. \\ benchohra@yahoo.com
}

\author{
OMAR BenNihi \\ Département de \\ mathématiques et \\ informatique, \\ Université de Saida, \\ 20000, Saida, Algérie. \\ obennihidz@yahoo.fr
}

\author{
KhaLiL EzZinBi \\ Département de \\ Mathématiques, \\ Faculté des Sciences, \\ Semlalia, B.P.2390, \\ Marrakech, Morocco. \\ ezzinbi@ucam. ac.ma
}

\begin{abstract}
In this paper we provide sufficient conditions for the existence and uniqueness of mild solutions for a class of neutral partial functional differential equations of fractional order with state-dependent delay. The nonlinear alternative of Frigon-Granas type for contractions maps in Fréchet spaces combined with $\alpha$-resolvent family is the main tool in our analysis.
\end{abstract}

\section{RESUMEN}

En este artículo entregamos condiciones suficientes para la existencia y unicidad de soluciones mild para una clase de ecuaciones diferenciales funcionales parciales neutrales de orden fraccionario con retraso dependiente del estado. La alternativa no lineal de tipo Frigon-Granas para contracciones en espacios de Féchet combinados con familias $\alpha$-resolvente es la herramienta principal en nuestro análisis.

Keywords and Phrases: Neutral functional differential equations; Riemman-Liouville fractional derivative; mild solution; delay; fixed point; $\alpha$-resolvent; Fréchet space.

2010 AMS Mathematics Subject Classification: 26A33; 34A08; 34K37. 


\section{Introduction}

In recent years fractional calculus has found many applications in physics, mechanics, chemistry, porous media, viscoelasticity, electrochemistry, electromagnetism, engineering, control, etc. [11, 24, 34, 37. Recent developments of differential and integral equations of fractional order are reported in the books by Abbas et al. [1, Baleanu et al. [11, Kilbas et al. [27, Lakshmikantham et al. 28], and the references therein. In this work, we discuss the existence of the unique mild solution defined on the semi-infinite positive real interval $[0,+\infty)$ for a class of neutral partial functional differential evolution equations of fractional order with state dependent delay. Complicated situations in which the delay depends on the unknown functions have been proposed in modeling in recent years. These equations are frequently called equations with state-dependent delay. Existence results and among other things were derived recently from functional differential equations when the solution is depending on the delay. We refer the reader to the papers by Adimy and Ezzinbi [2], Adimy et al. 3], 4, Agarwal et al. [5, 6, Aissani and Benchohra 7, Ait Dads and Ezzinbi [8], Dos Santos et al [17, and Hernandez et al. 23]. Over the past several years it has become apparent that equations with state-dependent delay arise also in several areas such as in classical electrodynamics [18, in population models [12, in models of commodity price fluctuations [13, 31, and in models of blood cell productions [32]. Recently Li and Peng [30] studied a class of abstract homogeneous fractional evolution equation. Baghli et al. [10, have proved global existence and uniqueness results for an initial value problem for functional differential equations of first order with state-dependent delay. Functional differential equations involving the Riemann-Liouville fractional derivative were considered by Benchohra et al. [14]. N'Guérékata and Mophou [33] studied semi linear neutral fractional functional evolution equations with infinite delay using the notion of $\alpha$ - resolvent family. Several works was published on the existence of mild solutions for this type of problems using different approaches and techniques like the approach of probability density function given by El Borai [19] and developed by Zhou and Jiao [41]. One can see also the work by Darwish and Ntouyas [15]. Recently Velusamy et al [38, studied the same problem using approach and technics based on the nonlinear alternative of Leray-Schauder. Motivated by the above papers, in this paper we studied the existence and uniqueness of solutions for neutral partial functional differential equations of fractional order with state-dependent delay in a real Banach space ( $E,||$. when the delay is infinite. Our contribution is to introduce a new approach based on the notion of semi norms in Fréchet spaces. In particular, we consider the following initial value problem

$$
\begin{aligned}
D^{\alpha}\left[x(t)-g\left(t, x_{\rho\left(t, x_{t}\right)}\right)\right]= & A x(t)+f\left(t, x_{\rho\left(t, x_{t}\right)}\right), \text { a.e. } t \in[0,+\infty), 0<\alpha<1, \\
& x_{0}=\varphi, \quad \varphi \in \mathcal{B},
\end{aligned}
$$

where $A: D(A) \subset E \rightarrow E$ is the infinitesimal generator of an $\alpha$-resolvent family $\left(T_{\alpha}(t)\right)_{t \geq 0}$ defined on a real Banach space $E, D^{\alpha}$ is understood here in the Riemann-Liouville sense, $f: J \times \mathcal{B} \rightarrow E$, $\rho: J \times \mathcal{B} \rightarrow \mathbb{R}$ and $\mathrm{g}: \mathrm{J} \times \mathcal{B} \rightarrow \mathrm{E}$ are appropriate given functions and satisfy some conditions that will be specified later, $\varphi$ belongs to an abstract space denoted $\mathcal{B}$ and called phase space with $\varphi(0)-g(0, \varphi)=0$. For any function $x$ defined on $(-\infty,+\infty)$ and any $t \in J$, we denote by $x_{t}$ the 
element of $\mathcal{B}$ defined by

$$
x_{t}(\theta)=x(t+\theta), \theta \in(-\infty, 0] .
$$

The function $x_{t}$ represents the history of the state from $-\infty$ up to the present time $t$. Our approach is based on the nonlinear alternative of Leray-Schauder type due to Frigon and Granas [21. These results can be considered as a contribution to this emerging field.

This paper is arranged as follows. In Section 2, some preliminary results are introduced. The main results are presented in Section 3, and in Section 4, an example is given to illustrate the abstract theory.

\section{Preliminaries}

In this section, we collect a few auxiliary results which will be needed in the sequel.

Let $b>0$. By $C([0, b] ; E)$ we denote the Banach space of continuous functions from $[0, b]$ into $E$, normed by

$$
\|x\|_{\infty}=\sup _{t \in[0, b]}|x(t)| .
$$

$B(E)$ is the space of bounded linear operators from $E$ into $E$, with the usual supremum norm

$$
\|\mathrm{N}\|_{\mathrm{B}(\mathrm{E})}=\sup \{|\mathrm{N}(\mathrm{X})|:|x|=1\} .
$$

A measurable function $x:[0, b] \rightarrow E$ is Bochner integrable if and only if $|x|$ is Lebesgue integrable. Let $\mathrm{L}^{1}([0, \mathrm{~b}], \mathrm{E})$ denotes the Banach space of measurable functions $x:[0, b] \rightarrow E$ which are Bochner integrable normed by

$$
\|x\|_{\mathrm{L}^{1}}=\int_{0}^{\mathrm{b}}|x(\mathrm{t})| \mathrm{dt}
$$

(For the Bochner integral properties, see the classical monograph of Yosida [39]).

Definition 2.1. [14] The fractional primitive of order $\alpha>0$ of a function $h: \mathbb{R}^{+} \rightarrow \mathbb{R}$ is defined by

$$
I_{0}^{\alpha} h(t):=\frac{1}{\Gamma(\alpha)} \int_{0}^{t}(t-s)^{\alpha-1} h(s) d s,
$$

provided the right side exist point wise on $\mathbb{R}^{+} . \Gamma$ is the gamma function.

Definition 2.2. 14] The fractional derivative of order $\alpha>0$ of a function $h: \mathbb{R}^{+} \rightarrow \mathbb{R}$ is defined as follow

$$
D_{0}^{\alpha} h(t):=\frac{1}{\Gamma(1-\alpha)} \frac{d}{d t} \int_{0}^{t}(t-s)^{-\alpha} h(s) d s=\frac{d}{d t} I_{0}^{1-\alpha} h(t) .
$$

Definition 2.3. [33] The Laplace transform of a function $\mathrm{f} \in \mathrm{L}_{\mathrm{loc}}^{1}\left(\mathbb{R}^{+}, \mathrm{E}\right)$ is defined by

$$
\widehat{f}(\lambda):=\int_{0}^{\infty} e^{-\lambda t} f(t) d t, \operatorname{Re}(\lambda)>\omega
$$

if the integral is absolutely convergent for $\operatorname{Re}(\lambda)>\omega$.

In order to defined the mild solution of the considered problem, we recall the following definition 
Definition 2.4. 33. Let $\mathrm{A}$ be a closed and linear operator with Domain $\mathrm{D}(\mathrm{A})$ defined on a Banach space $\mathrm{E}$. We call $\mathrm{A}$ the generator of an $\alpha$-resolvent family or solution operator if there exists $\omega>0$ and a strongly continuous function $\mathrm{T}_{\alpha}: \mathbb{R}^{+} \rightarrow \mathrm{L}(\mathrm{E})$ such that

$$
\{\lambda: \operatorname{Re}(\lambda)>\omega\} \subset \rho(A),
$$

and

$$
\left(\lambda^{\alpha}-A\right)^{-1} x=\int_{0}^{\infty} e^{-\lambda t} T_{\alpha}(t) x d t, \quad \operatorname{Re}(\lambda)>\omega \text { and } x \in E .
$$

$\mathrm{T}_{\alpha}(\mathrm{t})$ is called the solution operator generated by $\mathrm{A}$.

Proposition 2.5. [29] Let $\mathrm{T}_{\alpha}(\mathrm{t}) \in \mathrm{L}(\mathrm{E})$ be the solution operator with generator $\mathrm{A}$. Then the following conditions are satisfied:

(1) $\mathrm{T}_{\alpha}(\mathrm{t})$ is strongly continuous for $\mathrm{t} \geq 0$ and $\mathrm{T}_{\alpha}(0)=\mathrm{I}$.

(2) $T_{\alpha}(t) D(A) \subset D(A)$ and $A T_{\alpha}(t) x=T_{\alpha}(t) A x$ for all $x \in D(A), t \geq 0$.

(3) For every $x \in \mathrm{D}(\mathrm{A})$ and $\mathrm{t} \geq 0$, one has

$$
\mathrm{T}_{\alpha}(\mathrm{t}) x=x+\int_{0}^{\mathrm{t}} \frac{(\mathrm{t}-\mathrm{s})^{\alpha-1}}{\Gamma(\alpha)} \mathrm{AT} \mathrm{T}_{\alpha}(\mathrm{s}) x \mathrm{ds} .
$$

(4) Let $x \in \mathrm{D}(\mathrm{A})$. Then

$$
\int_{0}^{t} \frac{(t-s)^{\alpha-1}}{\Gamma(\alpha)} T_{\alpha}(s) x d s \in D(A)
$$

and

$$
\mathrm{T}_{\alpha}(\mathrm{t}) x=x+A \int_{0}^{\mathrm{t}} \frac{(\mathrm{t}-\mathrm{s})^{\alpha-1}}{\Gamma(\alpha)} \mathrm{T}_{\alpha}(\mathrm{s}) \mathrm{xds}
$$

Remark 2.6. The concept of a solution operator, as defined above, is closely related to the concept of a resolvent family (see Prüss)[35]. Because of the uniqueness of the Laplace transform, in the border case $\alpha=1$, the family $\mathrm{T}_{\alpha}(\mathrm{t})$ corresponds to the $\mathrm{C}_{0}$ - semigroup (see [20]), whereas in the case $\alpha=2$ a solution operator corresponds to the concept of cosine family (see [9]).

For more details on $\alpha$-resolvent family, we refer to [33] and the references therein. We will define the phase space $\mathcal{B}$ axiomatically, using ideas and notations developed by Hale and Kato [22]. (See also Kapper and Chappacher [26] and Schumacher [36]). More precisely, $\mathcal{B}$ will denote the vector space of functions defined from $(-\infty, 0]$ into $E$ endowed with a semi norm denoted $\|\cdot\|_{\mathcal{B}}$ and such that the following axioms hold.

- $\left(A_{1}\right)$ If $x:(-\infty, b) \rightarrow E$, is continuous on $[0, b]$ and $x_{0} \in \mathcal{B}$, then for $t \in[0, b)$ the following conditions hold 
- (i) $x_{t} \in \mathcal{B}$

- (ii) $\left\|x_{\mathrm{t}}\right\|_{\mathcal{B}} \leq \mathrm{K}(\mathrm{t}) \sup \{|x(\mathrm{~s})|: 0 \leq \mathrm{s} \leq \mathrm{t}\}+\mathrm{M}(\mathrm{t})\left\|\mathrm{x}_{0}\right\|_{\mathcal{B}}$,

$-\left(\right.$ iii) $|x(\mathrm{t})| \leq \mathrm{H}\left\|\mathrm{x}_{\mathrm{t}}\right\|_{\mathcal{B}}$

where $\mathrm{H} \geq 0$ is a constant, $\mathrm{K}:[0, \mathrm{~b}) \rightarrow[0,+\infty)$,

$M:[0,+\infty) \rightarrow[0,+\infty)$ with $K$ continuous and $M$ locally bounded and $H, K$ and $M$ are independent of $x($.$) .$

- $\left(A_{2}\right)$ For the function $x$ in $\left(A_{1}\right)$, the function $t \rightarrow x_{t}$ is a $\mathcal{B}$-valued continuous function on $[0, b]$.

- $\left(A_{3}\right)$ The space $\mathcal{B}$ is complete.

Denote $\mathrm{K}_{\mathrm{b}}=\sup \{\mathrm{K}(\mathrm{t}): \mathrm{t} \in[0, \mathrm{~b}]\}$ and $\mathrm{M}_{\mathrm{b}}=\sup \{\mathrm{M}(\mathrm{t}): \mathrm{t} \in[0, \mathrm{~b}]\}$.

Remark 2.7. (1) $[(i i i)]$ is equivalent to $|\phi(0)| \leq \mathrm{H}\|\phi\|_{\mathcal{B}}$ for every $\phi \in \mathcal{B}$.

(2) Since $\|\cdot\|_{\mathcal{B}}$ is a semi norm, two elements $\phi, \psi \in \mathcal{B}$ can verify $\|\phi-\psi\|_{\mathcal{B}}=0$ without necessarily $\phi(\theta)=\psi(\theta)$ for all $\theta \leq 0$.

(3) From the equivalence of in the first remark, we can see that for all $\phi, \psi \in \mathcal{B}$ such that $\|\phi-\psi\|_{\mathcal{B}}=0$, we necessarily have that $\phi(0)=\psi(0)$.

We now indicate some examples of phase spaces. For other details we refer, for instance to the book by Hino et al. 25].

Example 2.8. Let:

$\mathrm{BC}$ the space of bounded continuous functions defined from $(-\infty, 0]$ to $\mathrm{E}$;

BUC the space of bounded uniformly continuous functions defined from $(-\infty, 0]$ to $\mathrm{E}$;

$\mathrm{C}^{\infty}:=\left\{\phi \in \mathrm{BC}: \lim _{\theta \rightarrow-\infty} \phi(\theta)\right.$ exist in $\left.\mathrm{E}\right\}$

$C^{0}:=\left\{\phi \in B C: \lim _{\theta \rightarrow-\infty} \phi(\theta)=0\right\}$, endowed with the uniform norm

$$
\|\phi\|=\sup \{|\phi(\theta)|: \theta \leq 0\} .
$$

We have that the spaces BUC, $C^{\infty}$ and $C^{0}$ satisfy conditions $\left(A_{1}\right)-\left(A_{3}\right)$. However, $B C$ satisfies $\left(A_{1}\right),\left(A_{3}\right)$ but $\left(A_{2}\right)$ is not satisfied.

Example 2.9. The spaces $\mathrm{C}_{\mathrm{g}}, \mathrm{UC}_{\mathrm{g}}, \mathrm{C}_{\mathrm{g}}^{\infty}$ and $\mathrm{C}_{\mathrm{g}}^{0}$.

Let $\mathrm{g}$ be a positive continuous function on $(-\infty, 0]$. We define:

$$
C_{g}:=\left\{\phi \in C((-\infty, 0], E): \frac{\phi(\theta)}{g(\theta)} \text { is bounded on }(-\infty, 0]\right\} ;
$$




$$
\begin{gathered}
C_{g}^{0}:=\left\{\phi \in C_{g}: \lim _{\theta \rightarrow-\infty} \frac{\phi(\theta)}{g(\theta)}=0\right\}, \text { endowed with the uniform norm } \\
\|\phi\|=\sup \left\{\frac{|\phi(\theta)|}{g(\theta)}: \theta \leq 0\right\} .
\end{gathered}
$$

Then we have that the spaces $C_{g}$ and $C_{g}^{0}$ satisfy conditions $\left(A_{1}\right)-\left(A_{3}\right)$. We consider the following condition on the function $\mathrm{g}$.

$\left(g_{1}\right)$ For all $\mathrm{a}>0, \sup _{0 \leq \mathrm{t} \leq \mathrm{a}} \sup \left\{\frac{\mathrm{g}(\mathrm{t}+\theta)}{\mathrm{g}(\theta)}:-\infty<\theta \leq-\mathrm{t}\right\}<\infty$.

They satisfy conditions $\left(\mathrm{A}_{1}\right)$ and $\left(\mathrm{A}_{2}\right)$ if $\left(\mathrm{g}_{1}\right)$ holds.

Example 2.10. The space $\mathrm{C}_{\gamma}$. For any real constant $\gamma$, we define the functional space $\mathrm{C}_{\gamma}$ by

$$
\mathrm{C}_{\gamma}:=\left\{\phi \in \mathrm{C}((-\infty, 0], \mathrm{E}): \lim _{\theta \rightarrow-\infty} \mathrm{e}^{\gamma \theta} \phi(\theta) \text { exists in } \mathrm{E}\right\}
$$

endowed with the following norm

$$
\|\phi\|=\sup \left\{e^{\gamma \theta}|\phi(\theta)|: \theta \leq 0\right\} .
$$

Then $\mathrm{C}_{\gamma}$ satisfies axioms $\left(\mathrm{A}_{1}\right)-\left(\mathrm{A}_{3}\right)$.

Let $E=\left(E,\|\cdot\|_{\mathfrak{n}}\right)$ be a Fréchet space with a family of semi-norms $\left\{\|\cdot\|_{\mathfrak{n}}\right\}_{\mathfrak{n} \in \mathbb{N}}$. We say that $X$ is bounded if for every $n \in \mathbb{N}$, there exists $\bar{M}_{n}>0$ such that

$$
\|x\|_{n} \leq \bar{M}_{n} \quad \text { for all } x \in X
$$

To $E$ we associate a sequence of Banach spaces $\left\{\left(E^{n},\|\cdot\|_{n}\right)\right\}$ as follows: For every $n \in \mathbb{N}$, we consider the equivalence relation $\sim_{n}$ defined by $: x \sim_{n} y$ if and only if $\|x-y\|_{n}=0$ for $x, y \in E$. We denote $E^{n}=\left(\left.E\right|_{\sim_{n}},\|\cdot\|_{n}\right)$ the quotient space, and we set $\left(E^{n},\|\cdot\|_{n}\right)$ the completion of $E^{n}$ with respect to $\|\cdot\|_{n}$. To every $X \subset E$, we associate a sequence $\left\{X^{n}\right\}$ of subsets $X^{n} \subset E^{n}$ as follows: For every $x \in E$, we denote $[x]_{n}$ the equivalence class of $x$ in $E^{n}$ and we define $X^{n}=\left\{[x]_{n}: x \in X\right\}$. We denote $\overline{X^{n}}$, int $t_{n}\left(X^{n}\right)$ and $\partial_{n} X^{n}$, respectively, the closure, the interior and the boundary of $X^{n}$ with respect to $\|\cdot\|_{n}$ in $E^{n}$.

We assume that the family of semi-norms $\left\{\|\cdot\|_{\mathfrak{n}}\right\}_{\mathfrak{n} \in \mathbb{N}}$ verifies:

$$
\|x\|_{1} \leq\|x\|_{2} \leq\|x\|_{3} \leq \ldots \text { for every } x \in X
$$

The following definition is the appropriate concept of contraction in E.

Definition 2.11. 211] A function $\mathrm{f}: \mathrm{E} \rightarrow \mathrm{E}$ is said to be a contraction if for every $\mathrm{n} \in \mathbb{N}$ there exists $\mathrm{k}_{\mathrm{n}} \in[0,1)$ such that:

$$
\|f(x)-f(y)\|_{n} \leq k_{n}\|x-y\|_{n} \text { for all } x, y \in E .
$$


The corresponding nonlinear alternative result is as follows:

Theorem 2.12. (Nonlinear Alternative) [21]. Let $\mathrm{E}$ be a Fréchet space and $\mathrm{X}$ be a closed subset of $\mathrm{E}$ such that $\mathrm{O} \in \mathrm{X}$ and let $\mathrm{N}: \mathrm{X} \rightarrow \mathrm{E}$ be a contraction map such that $\mathrm{N}(\mathrm{X})$ is bounded. Then one of the following statements holds:

$\left(S_{1}\right) \mathrm{N}$ has a unique fixed point in $\mathrm{E}$.

$\left(\mathrm{S}_{2}\right)$ There exist $0 \leq \lambda<1, \mathrm{n} \in \mathbb{N}$ and $\mathrm{x} \in \partial_{\mathrm{n}} \mathrm{X}^{\mathrm{n}}$ such that $\|\mathrm{x}-\lambda \mathrm{N}(\mathrm{x})\|_{\mathrm{n}}=0$.

\section{3 main results}

Before starting and proving the main results, let us give the definition of mild solution to the neutral partial evolution problem (11)-(2). Throughout this work, the function $f: J \times \mathcal{B} \rightarrow E$ will be continuous.

Definition 3.1. A function $\mathrm{x}$ is said to be a mild solution of (11)-(2) if $\mathrm{x}$ satisfies

$$
x(t)=\left\{\begin{array}{l}
\varphi(t), \quad t \in(-\infty, 0], \\
g\left(t, x_{\rho\left(t, x_{t}\right)}\right)+\int_{0}^{t} T_{\alpha}(t-s) A(s) g\left(s, x_{\rho\left(s, x_{s}\right)}\right) d s \\
+\int_{0}^{t} T_{\alpha}(t-s) f\left(s, x_{\rho\left(s, x_{s}\right)}\right) d s, \quad t \in J .
\end{array}\right.
$$

Set $\mathcal{R}\left(\rho^{-}\right)=\{\rho(s, \varphi):(s, \varphi) \in \mathrm{J} \times \mathcal{B}, \rho(s, \varphi) \leq 0\}$. We always assume that $\rho: \mathrm{J} \times \mathcal{B} \rightarrow \mathbb{R}$ is continuous.

Let $\widehat{M}$ be such that $\widehat{M}=\sup _{t \in J}\left|T_{\alpha}(t)\right|$ then

$$
\left\|\mathrm{T}_{\alpha}(\mathrm{t})\right\|_{\mathrm{B}(\mathrm{E})} \leq \widehat{\mathrm{M}}, \quad \mathrm{t} \in \mathrm{J} .
$$

Additionally, we will need to introduce the following hypotheses which are assumed thereafter:

$\left(\mathrm{H}_{\varphi}\right)$ The function $t \rightarrow \varphi_{\mathrm{t}}$ is continuous from $\mathcal{R}\left(\rho^{-}\right)$into $\mathcal{B}$ and there exists a continuous and bounded function $\mathrm{L}^{\varphi}: \mathcal{R}\left(\rho^{-}\right) \rightarrow(0, \infty)$ such that

$$
\left\|\varphi_{\mathrm{t}}\right\|_{\mathcal{B}} \leq \mathrm{L}^{\varphi}(\mathrm{t})\|\varphi\|_{\mathcal{B}} \quad \text { for every } \mathrm{t} \in \mathcal{R}\left(\rho^{-}\right)
$$

Remark 3.2. The condition $\left(\mathrm{H}_{\varphi}\right)$, is frequently verified by continuous and bounded functions. For more details, see for instance ([25], Proposition 7.1.1).

$\left(\mathrm{H}_{1}\right)$ There exist a function $p \in \mathrm{L}_{\mathrm{loc}}^{1}\left(\mathrm{~J}, \mathbb{R}^{+}\right)$and a continuous nondecreasing function $\psi:[0,+\infty) \rightarrow$ $(0, \infty)$ such that

$$
|\mathbf{f}(\mathrm{t}, \mathrm{u})| \leq \mathrm{p}(\mathrm{t}) \psi\left(\|\boldsymbol{u}\|_{\mathcal{B}}\right) \text { for a.e. } \mathrm{t} \in \mathrm{J} \text { and each } \boldsymbol{u} \in \mathcal{B} \text {. }
$$


$\left(\mathrm{H}_{2}\right)$ For all $n>0$, there exists $\mathrm{l}_{n} \in \mathrm{L}_{\mathrm{loc}}^{1}\left(\mathrm{~J}, \mathbb{R}^{+}\right)$such that:

$$
|f(t, u)-f(t, v)| \leq l_{n}(t)\|u-v\|_{\mathcal{B}} \text { for all } t \in[0, n] \text { and } u, v \in \mathcal{B} \text {. }
$$

$\left(\mathrm{H}_{3}\right)$ There exists a constant $\bar{M}_{0}>0$ such that

$$
\left\|A^{-1}(t)\right\|_{B(E)} \leq \bar{M}_{0} \quad \text { for all } t \in J .
$$

$\left(\mathrm{H}_{4}\right)$ There exists a constant $\mathrm{L}_{*}>0$ such that

$$
|A(s) g(s, \varphi)-A(\bar{s}) g(\bar{s}, \bar{\varphi})| \leq L_{*}\left(|s-\bar{s}|+\|\varphi-\bar{\varphi}\|_{\mathcal{B}}\right)
$$

for all $s, \bar{s} \in \mathrm{J}$ and $\varphi, \bar{\varphi} \in \mathcal{B}$.

Consider the following space

$$
\mathrm{B}_{+\infty}=\left\{x: \mathbb{R} \rightarrow \mathrm{E}:\left.x\right|_{[0, b]} \text { continuous for } \mathrm{b}>0 \text { and } x_{0} \in \mathcal{B}\right\},
$$

where $\left.x\right|_{[0, b]}$ is the restriction of $x$ to the real compact interval $[0, b]$.

Let us fix $r>1$. For every $n \in \mathbb{N}$, we define in $B_{+\infty}$ the semi norms by:

$$
\|x\|_{n}:=\sup \left\{e^{-r L_{n}^{*}(t)}|x(t)|: t \in[0, n]\right\}
$$

where $L_{n}^{*}(t)=\int_{0}^{t} \bar{l}_{n}(s) d s, \bar{l}_{n}(t)=K_{n} \widehat{M} l_{n}(t)$ and $l_{n}$ is the function given in $\left(H_{2}\right)$.

Then $B_{+\infty}$ is a Fréchet space with those semi norms family $\|\cdot\|_{n}$.

Lemma 3.3. [23], (Lemma 2.4) If $\mathrm{x}:(-\infty, \mathrm{b}] \rightarrow \mathrm{E}$ is a function such that $\mathrm{x}_{0}=\varphi$, then

$$
\left\|x_{s}\right\|_{\mathcal{B}} \leq\left(M_{b}+L^{\varphi}\right)\|\varphi\|_{\mathcal{B}}+K_{b} \sup \{|x(\theta)|, \theta \in[0, \max (0, s)]\}, s \in \mathcal{R}\left(\rho^{-}\right) \cup \mathrm{J}
$$

where $\mathrm{L}^{\varphi}=\sup _{t \in \mathcal{R}\left(\rho^{-}\right)} \mathrm{L}^{\varphi}(\mathrm{t})$.

Theorem 3.4. Suppose the hypothesis $\left(\mathrm{H}_{\varphi}\right)$ and $\left(\mathrm{H}_{1}\right)-\left(\mathrm{H}_{4}\right)$ are satisfied and moreover for each $\mathrm{n} \in \mathbb{N}$

$$
\int_{\delta_{n}}^{+\infty} \frac{d s}{s+\psi(s)}>\frac{\widehat{M} K_{n}}{1-\bar{M}_{0} L K_{n}} \int_{0}^{n} \max (L, p(s)) d s
$$

with

$$
\delta_{n}=c_{n}+K_{n} L \frac{\bar{M}_{0}(1+\widehat{M})+\widehat{M} n+\bar{M}_{o}\left[c_{n}+\widehat{M}\|\varphi\|_{\mathcal{B}}\right]}{1-\bar{M}_{0} L K_{n}}
$$

and $\mathrm{c}_{\mathrm{n}}=\left(\mathrm{M}_{\mathrm{n}}+\mathrm{L}^{\varphi}+\mathrm{K}_{\mathrm{n}} \widehat{\mathrm{M}} \mathrm{H}\right)\|\varphi\|_{\mathcal{B}}$, then the problem (10) - (2) has a unique mild solution. 
Proof. Consider the operator $\mathrm{N}: \mathrm{B}_{+\infty} \rightarrow \mathrm{B}_{+\infty}$ defined by :

$$
N(x)(t)=\left\{\begin{array}{lr}
\varphi(t), & \text { if } t \leq 0 \\
g\left(t, x_{\rho\left(t, x_{t}\right)}\right) & \\
+\int_{0}^{t} T_{\alpha}(t-s) A(s) g\left(s, x_{\rho\left(s, x_{s}\right)}\right) d s & \\
+\int_{0}^{t} T_{\alpha}(t-s) f\left(s, x_{\rho\left(s, x_{s}\right)}\right) d s, & \text { if } t \in J .
\end{array}\right.
$$

Then, fixed points of the operator $\mathrm{N}$ are mild solutions of the problem (11) - (2).

For $\varphi \in \mathcal{B}$, we consider the function $x():. \mathbb{R} \rightarrow E$ defined as bellow by

$$
y(t)= \begin{cases}\varphi(t), & \text { if } t \leq 0 \\ 0, & \text { if } t \in J\end{cases}
$$

Then $y_{0}=\varphi$. For each function $z \in \mathrm{B}_{+\infty}$ with $z(0)=0$, we consider the function $\bar{z}$ by

$$
\bar{z}(t)= \begin{cases}0, & \text { if } t \leq 0 \\ z(t), & \text { if } t \in J\end{cases}
$$

If $x(\cdot)$ satisfies (3.1), we decompose it as $x(t)=z(t)+y(t), t \geq 0$, which implies $x_{t}=z_{t}+y_{t}$, for every $\mathrm{t} \in \mathrm{J}$ and the function $z(\cdot)$ satisfies $z_{0}=0$ and for $\mathrm{t} \in \mathrm{J}$, we get

$$
\begin{aligned}
z(t) & =g\left(t, z_{\rho\left(t, z_{t}+y_{t}\right)}+y_{\rho\left(t, z_{t}+y_{t}\right)}\right) \\
& +\int_{0}^{t} T_{\alpha}(t-s) A(s) g\left(s, z_{\rho\left(s, z_{s}+y_{s}\right)}+y_{\rho\left(s, z_{s}+y_{s}\right)}\right) d s \\
& +\int_{0}^{t} T_{\alpha}(t-s) f\left(s, z_{\rho\left(s, z_{s}+y_{s}\right)}+y_{\rho\left(s, z_{s}+y_{s}\right)}\right) d s .
\end{aligned}
$$

Define the operator $\mathrm{F}: \mathrm{B}_{+\infty}^{0} \rightarrow \mathrm{B}_{+\infty}^{0}$ by :

$$
\begin{aligned}
F(z)(t) & =g\left(t, z_{\rho\left(t, z_{s}+y_{s}\right)}+y_{\rho\left(t, z_{s}+y_{s}\right)}\right) \\
& +\int_{0}^{t} T_{\alpha}(t-s) A(s) g\left(s, z_{\rho\left(s, z_{s}+y_{s}\right)}+y_{\rho\left(s, z_{s}+y_{s}\right)}\right) d s \\
& +\int_{0}^{t} T_{\alpha}(t-s) f\left(s, z_{\rho\left(s, z_{s}+y_{s}\right)}+y_{\rho\left(s, z_{s}+y_{s}\right)}\right) d s .
\end{aligned}
$$

Obviously the operator $\mathrm{N}$ has a fixed point is equivalent to $\mathrm{F}$ has one, so it turns to prove that $\mathrm{F}$ has a fixed point. Let $z \in \mathrm{B}_{+\infty}^{0}$ be such that $z=\lambda \mathrm{F}(z)$ for some $\lambda \in[0,1)$. Then, using $(\mathrm{H} 1)-(\mathrm{H} 4)$, 
we have for each $t \in[0, n]$

$$
\begin{aligned}
& |z(t)| \leq\left|g\left(t, z_{\rho\left(t, z_{t}+y_{t}\right)}+y_{\rho\left(t, z_{t}+y_{t}\right)}\right)\right| \\
& +\left|\int_{0}^{t} T_{\alpha}(t-s) A(s) g\left(s, z_{\rho\left(s, z_{s}+y_{s}\right)}+y_{\rho\left(s, z_{s}+y_{s}\right)}\right) d s\right| \\
& +\left|\int_{0}^{t} T_{\alpha}(t-s) f\left(s, z_{\rho\left(s, z_{s}+y_{s}\right)}+y_{\rho\left(s, z_{s}+y_{s}\right)}\right) d s\right| \\
& \leq\left\|A^{-1}(t)\right\|_{B(E)}\left\|A(t) g\left(t, z_{\rho\left(t, z_{t}+y_{t}\right)}+y_{\rho\left(t, z_{t}+y_{t}\right)}\right)\right\| \\
& +\int_{0}^{t}\left\|T_{\alpha}(t-s)\right\|_{B(E)}\left\|A(s) g\left(s, z_{\rho\left(s, z_{s}+y_{s}\right)}+y_{\rho\left(s, z_{s}+y_{s}\right)}\right)\right\| d s \\
& +\int_{0}^{t}\left\|T_{\alpha}(t-s)\right\|_{B(E)}\left|f\left(s, z_{\rho\left(s, z_{s}+y_{s}\right)}+y_{\rho\left(s, z_{s}+y_{s}\right)}\right)\right| d s \\
& \leq \bar{M}_{0} \mathrm{~L}\left(\left\|z_{\rho\left(t, z_{s}+y_{s}\right)}+y_{\rho\left(t, z_{s}+y_{s}\right)}\right\|_{\mathcal{B}}+1\right)+\widehat{M} \bar{M}_{0} \mathrm{~L}\left(\|\varphi\|_{\mathcal{B}}+1\right) \\
& +\widehat{M} \int_{0}^{t} \mathrm{~L}\left(\left\|z_{\rho\left(s, z_{s}+y_{s}\right)}+y_{\rho\left(s, z_{s}+y_{s}\right)}\right\|_{\mathcal{B}}+1\right) d s \\
& +\widehat{M} \int_{0}^{t} p(s) \psi\left(\left\|z_{\rho\left(s, z_{s}+y_{s}\right)}+y_{\rho\left(s, z_{s}+y_{s}\right)}\right\|_{\mathcal{B}}\right) d s \\
& \leq \bar{M}_{0} L\left\|z_{\rho\left(t, z_{t}+y_{t}\right)}+y_{\rho\left(t, z_{t}+y_{t}\right)}\right\|_{\mathcal{B}}+\bar{M}_{0} \mathrm{~L}(1+\widehat{M})+\widehat{M} \mathrm{Ln}+\widehat{M} \bar{M}_{0} \mathrm{~L}\|\varphi\|_{\mathcal{B}} \\
& +\widehat{M} L \int_{0}^{t}\left\|z_{\rho\left(s, z_{s}+y_{s}\right)}+y_{\rho\left(s, z_{s}+y_{s}\right)}\right\|_{\mathcal{B}} d s \\
& +\widehat{M} \int_{0}^{t} p(s) \psi\left(\left\|z_{\rho\left(s, z_{s}+y_{s}\right)}+y_{\rho\left(s, z_{s}+y_{s}\right)}\right\|_{\mathcal{B}}\right) d s .
\end{aligned}
$$

Using the assumption $A_{1}$, we get

$$
\begin{aligned}
\left\|z_{\rho\left(t, z_{s}+y_{s}\right)}+y_{\rho\left(t, z_{s}+y_{s}\right)}\right\|_{\mathcal{B}} & \leq\left\|z_{\rho\left(t, z_{s}+y_{s}\right)}\right\|_{\mathcal{B}}+\left\|y_{\rho\left(t, z_{s}+y_{s}\right)}\right\|_{\mathcal{B}} \\
& \leq K(s)|z(s)|+M(s)\left\|Z_{0}\right\|_{\mathcal{B}}+K(s)|y(s)|+M(s)\left\|y_{0}\right\|_{\mathcal{B}} \\
& \leq K_{n}|z(s)|+M_{n} M|\varphi(0)|+M n\|\varphi\|_{\mathcal{B}} \\
& \leq K_{n}|z(s)|+M_{n} M H\|\varphi\|_{\mathcal{B}}+M_{n}\|\varphi\|_{\mathcal{B}} \\
& \leq K_{n}|z(s)|+\left(K_{n} M H+M_{n}\right)\|\varphi\|_{\mathcal{B}} .
\end{aligned}
$$

Set $c_{n}=\left(K_{n} M H+M_{n}\right)\|\varphi\|_{\mathcal{B}}$ we obtain

$$
\begin{aligned}
|z(t)| & \leq \bar{M}_{0} L\left(K_{n}|z(t)|+c_{n}\right)+\bar{M}_{0} L(1+\widehat{M})+\widehat{M} L n+\widehat{M} \bar{M}_{0} L\|\varphi\|_{\mathcal{B}} \\
& +\widehat{M} L \int_{0}^{t}\left(K_{n}|z(s)|+c_{n}\right) d s+\widehat{M} \int_{0}^{t} p(s) \psi\left(K_{n}|z(s)|+c_{n}\right) d s \\
& \leq \bar{M}_{0} L K_{n}|z(t)|+\bar{M}_{0} L(1+\widehat{M})+\widehat{M} L n+\bar{M}_{0} L c_{n}+\widehat{M} \bar{M}_{0} L\|\varphi\|_{\mathcal{B}} \\
& +\widehat{M} L \int_{0}^{t}\left(K_{n}|z(s)|+c_{n}\right) d s+\widehat{M} \int_{0}^{t} p(s) \psi\left(K_{n}|z(s)|+c_{n}\right) d s .
\end{aligned}
$$

Then 


$$
\begin{aligned}
\left(1-\bar{M}_{0} L K n\right)|z(t)| & \leq \mathrm{L}\left(\bar{M}_{0}(1+\widehat{M})+\widehat{M} n+\bar{M}_{0} c_{n}+\widehat{M} \bar{M}_{0}\|\varphi\|_{\mathcal{B}}\right) \\
& +\widehat{M} L \int_{0}^{t}\left(K_{n}|z(s)|+c_{n}\right) d s+\widehat{M} \int_{0}^{t} p(s) \psi\left(K_{n}|z(s)|+c_{n}\right) d s
\end{aligned}
$$

Set

$$
\delta_{n}:=c_{n}+\frac{L_{n}}{1-\bar{M}_{0} L K_{n}}\left[\bar{M}_{0}(1+\widehat{M})+\widehat{M} n+\bar{M}_{0} c_{n}+\widehat{M} \bar{M}_{0}\|\varphi\|_{\mathcal{B}}\right]
$$

Thus

$$
\begin{aligned}
K_{n}|z(t)|+c_{n} & \leq \delta_{n}+\frac{\widehat{M} L K_{n}}{1-\bar{M}_{0} L K_{n}} \int_{0}^{t}\left(K_{n}|z(s)|+c_{n}\right) d s \\
& +\frac{\widehat{M} K_{n}}{1-\bar{M}_{0} L K_{n}} \int_{0}^{t} p(s) \psi\left(K_{n}|z(s)|+c_{n}\right) d s .
\end{aligned}
$$

We consider the function $\mu$ defined by

$$
\mu(\mathrm{t}):=\sup \left\{\mathrm{K}_{\mathrm{n}}|z(\mathrm{~s})|+\mathrm{c}_{\mathrm{n}}: 0 \leq \mathrm{s} \leq \mathrm{t}\right\}, \quad 0 \leq \mathrm{t}<+\infty .
$$

Let $t^{\star} \in[0, t]$ be such that $\mu\left(t^{\star}\right)=K_{n}\left|z\left(t^{\star}\right)\right|+c_{n}$. By the previous inequality, we have

$$
\mu(t) \leq \delta_{n}+\frac{\widehat{M} K_{n}}{1-\bar{M}_{0} L K_{n}}\left[\int_{0}^{t} L \mu(s) d s+\int_{0}^{t} p(s) \psi(\mu(s)) d s\right] \quad \text { for } t \in[0, n] .
$$

Let us take the right-hand side of the above inequality as $v(t)$. Then, we have

$$
\mu(t) \leq v(t) \text { for all } t \in[0, n] .
$$

From the definition of $v$, we have $v(0)=\delta_{n}$ and

$$
v^{\prime}(t)=\frac{\widehat{M} K_{n}}{1-\bar{M}_{0} L K_{n}}[L \mu(t)+p(t) \psi(\mu(t))] \quad \text { a.e. } t \in[0, n] .
$$

Using the nondecreasing character of $\psi$, we get

$$
v^{\prime}(t) \leq \frac{\widehat{M} K_{n}}{1-\bar{M}_{0} L K_{n}}[L(t)+p(t) \psi(v(t))] \quad \text { a.e. } t \in[0, n] .
$$

Using the condition [6], this implies that for each $t \in[0, n]$, we have

$$
\begin{aligned}
\int_{\delta_{n}}^{v(t)} \frac{d s}{s+\psi(s)} & \leq \frac{\widehat{M} K_{n}}{1-\bar{M}_{0} L K_{n}} \int_{0}^{t} \max (L, p(s)) d s \\
& \leq \frac{\widehat{M} K_{n}}{1-\bar{M}_{0} L K_{n}} \int_{0}^{n} \max (L, p(s)) d s \\
& <\int_{\delta_{n}}^{+\infty} \frac{d s}{s+\psi(s)} .
\end{aligned}
$$


Thus, for every $t \in[0, n]$, there exists a constant $\Lambda_{n}$ such that $v(t) \leq \Lambda_{n}$ and hence $\mu(t) \leq \Lambda_{n}$. Since $\|z\|_{n} \leq \mu(t)$, we have $\|z\|_{n} \leq \Lambda_{n}$.

Now, we shall show that $\mathrm{F}: \mathrm{Z} \rightarrow \mathrm{B}_{+\infty}^{0}$ is a contraction operator.

Indeed, consider $z, \bar{z} \in Z$, thus for each $\mathrm{t} \in[0, \mathrm{n}]$ and $\mathrm{n} \in \mathbb{N}$

$$
\begin{aligned}
& |F(z)(t)-F(\bar{z})(t)| \leq\left|g\left(t, z_{\rho\left(t, z_{t}+y_{t}\right)}+y_{\rho\left(t, z_{t}+y_{t}\right)}\right)-g\left(t, \bar{z}_{\rho\left(t, z_{t}+y_{t}\right)}+y_{\rho\left(t, z_{t}+y_{t}\right)}\right)\right| \\
+ & \int_{0}^{t}\left\|T_{\alpha}(t-s)\right\|_{B(E)}\left|A(s)\left[g\left(s, z_{\rho\left(s, z_{s}+y_{s}\right)}+y_{\rho\left(s, z_{s}+y_{s}\right)}\right)-g\left(s, \bar{z}_{\rho\left(s, z_{s}+y_{s}\right)}+y_{\rho\left(s, z_{s}+y_{s}\right)}\right)\right]\right| d s \\
+ & \int_{0}^{t}\left\|T_{\alpha}(t-s)\right\|_{B(E)}\left|f\left(s, z_{\rho\left(s, z_{s}+y_{s}\right)}+y_{\rho\left(s, z_{s}+y_{s}\right)}\right)-f\left(s, \bar{z}_{\rho\left(s, z_{s}+y_{s}\right)}+y_{\rho\left(s, z_{s}+y_{s}\right)}\right)\right| d s \\
\leq & \left\|A^{-1}(t)\right\|_{B(E)}\left|A(t) g\left(t, z_{\rho\left(t, z_{t}+y_{t}\right)}+y_{\rho\left(t, z_{t}+y_{t}\right)}\right)-A(t) g\left(t, \bar{z}_{\rho\left(t, z_{t}+y_{t}\right)}+y_{\rho\left(t, z_{t}+y_{t}\right)}\right)\right| \\
+ & \int_{0}^{t} \widehat{M}\left|A(s) g\left(s, z_{\rho\left(s, z_{s}+y_{s}\right)}+y_{\rho\left(s, z_{s}+y_{s}\right)}\right)-A(s) g\left(s, \bar{z}_{\rho\left(s, z_{s}+y_{s}\right)}+y_{\rho\left(s, z_{s}+y_{s}\right)}\right)\right| d s \\
+ & \int_{0}^{t} \widehat{M}\left|f\left(s, z_{\rho\left(s, z_{s}+y_{s}\right)}+y_{\rho\left(s, z_{s}+y_{s}\right)}\right)-f\left(s, \bar{z}_{\rho\left(s, z_{s}+y_{s}\right)}+y_{\rho\left(s, z_{s}+y_{s}\right)}\right)\right| d s \\
\leq & \bar{M}_{0} L_{\star}\left\|z_{\rho\left(t, z_{t}+y_{t}\right)}-\bar{z}_{\rho\left(t, z_{t}+y_{t}\right)}\right\|_{\mathcal{B}}+\int_{0}^{t} \widehat{M} L_{\star}\left\|z_{\rho\left(s, z_{s}+y_{s}\right)}-\bar{z}_{\rho\left(s, z_{s}+y_{s}\right)}\right\|_{\mathcal{B}} \mathrm{ds} \\
+ & \int_{0}^{t} \widehat{M} l_{n}(s)\left\|z_{\rho\left(s, z_{s}+y_{s}\right)}-\bar{z}_{\rho\left(s, z_{s}+y_{s}\right)}\right\|_{\mathcal{B}} \mathrm{ds} \\
\leq & \left.\bar{M}_{0} L_{\star}\left\|z_{\rho\left(t, z_{t}+y_{t}\right)}-\bar{z}_{\rho\left(t, z_{t}+y_{t}\right)}\right\|_{\mathcal{B}}+\int_{0}^{t} \widehat{M}\left[L_{\star}+l_{n}(s)\right] \| z_{\rho\left(s, z_{s}+y_{s}\right)}-\bar{z}_{\rho\left(s, z_{s}+y_{s}\right)}\right) \|_{\mathcal{B}} \mathrm{ds} .
\end{aligned}
$$

Since $\left\|z_{\rho\left(t, z_{\mathfrak{t}}+y_{\mathfrak{t}}\right)}\right\|_{\mathcal{B}} \leq K_{n}|z(t)|+c_{n}$ we obtain

$$
|F(z)(t)-F(\bar{z})(t)| \leq \bar{M}_{0} L_{*} K_{n}|z(t)-\bar{z}(t)|+\int_{0}^{t} \widehat{M}\left[L_{*}+l_{n}(s)\right] K_{n}|z(s)-\bar{z}(s)| d s .
$$

Let us take here $\bar{l}_{n}(t)=\widehat{M} K_{n}\left[L_{*}+l_{n}(t)\right]$ for the family semi norm $\left\{\|\cdot\|_{n}\right\}_{\mathfrak{n} \in \mathbb{N}}$, then

$$
\begin{aligned}
|F(z)(t)-F(\bar{z})(t)| & \leq \bar{M}_{0} L_{*} K_{n}|z(t)-\bar{z}(t)|+\int_{0}^{t} l_{n}^{-}(s)|z(s)-\bar{z}(s)| d s \\
& \leq\left[\bar{M}_{0} L_{*} K_{n} e^{\tau L_{n}^{*}(t)}\right]\left[e^{-\tau L_{n}^{*}(t)}|z(t)-\bar{z}(t)|\right] \\
& +\int_{0}^{t}\left[\bar{l}_{n}(s) e^{\tau L_{n}^{*}(s)}\right]\left[e^{-\tau L_{n}^{*}(s)}|z(s)-\bar{z}(s)|\right] d s \\
& \leq \bar{M}_{0} L_{*} K_{n} e^{\tau L_{n}^{*}(t)}\|z-\bar{z}\|_{n}+\int_{0}^{t}\left[\frac{e^{\tau L_{n}^{*}(s)}}{\tau}\right]^{\prime} d s\|z-\bar{z}\|_{n} \\
& \leq\left[\bar{M}_{0} L_{*} K_{n}+\frac{1}{\tau}\right] e^{\tau L_{n}^{*}(t)}\|z-\bar{z}\|_{n} .
\end{aligned}
$$

Therefore,

$$
\|\mathrm{F}(z)-\mathrm{F}(\bar{z})\|_{n} \leq\left[\bar{M}_{0} \mathrm{~L}_{*} \mathrm{~K}_{\mathrm{n}}+\frac{1}{\tau}\right]\|z-\bar{z}\|_{n} .
$$


So, for an appropriate choice of $L_{*}$ and $\tau$ such that

$$
\bar{M}_{0} L_{*} K_{n}+\frac{1}{\tau}<1,
$$

the operator $\mathrm{F}$ is a contraction for all $n \in \mathbb{N}$. From the choice of $Z$ there is no $z \in \partial Z^{n}$ such that $z=\lambda \mathrm{F}(z)$ for some $\lambda \in(0,1)$. Then the statement S2 in Theorem 2.12 does not hold. A consequence of the nonlinear alternative of Frigon and Granas shows that the statement S1 holds. We deduce that the operator $F$ has a unique fixed-point $z^{\star}$. Then $x^{\star}(t)=z^{\star}(t)+y^{\star}(t)$, $t \in(-\infty,+\infty)$ is a fixed point of the operator $N$, which is the unique mild solution of the problem (11) - (2).

\section{4 example}

To illustrate our results, we give an example

Example 4.1. Consider the neutral evolution equation

$$
\left\{\begin{array}{lr} 
& \frac{\partial^{\alpha}}{\partial \partial^{\alpha}}\left[u(t, \xi)-\int_{-\infty}^{0} a_{3}(s-t) u\left(s-\rho_{1}(t) \rho_{2}\left(\int_{0}^{\pi} a_{2}(\theta)|u(t, \theta)|^{2} d \theta\right), \xi\right) d s\right] \\
= & \frac{\partial^{2} u(t, \xi)}{\partial \xi^{2}}+a_{0}(t, \xi) u(t, \xi) \\
+\int_{-\infty}^{0} a_{1}(s-t) u\left(s-\rho_{1}(t) \rho_{2}\left(\int_{0}^{\pi} a_{2}(\theta)|u(t, \theta)|^{2} d \theta\right), \xi\right) d s, & t \geq 0, \xi \in[0, \pi], \\
v(t, 0)=v(t, \pi)=0, & t \geq 0, \\
v(\theta, \xi)=v_{0}(\theta, \xi), & -\infty<\theta \leq 0, \xi \in[0, \pi],
\end{array}\right.
$$

To represent this system in the abstract form (11)-(2), we choose the space

$\mathrm{E}=\mathrm{L}^{2}([0, \pi], \mathbb{R})$ and the operator $\mathrm{A}: \mathrm{D}(\mathrm{A}) \subset \mathrm{E} \rightarrow \mathrm{E}$ is given by $\mathrm{A} \omega=\omega^{\prime \prime}$ with domain

$$
D(A):=\left\{\omega \in E: \omega^{\prime \prime} \in E, \omega(0)=\omega(\pi)=0\right\} .
$$

It is well known that $\mathrm{A}$ is an infinitesimal generator of an $\alpha$-resolvent family $\left(\mathrm{T}_{\alpha}(\mathrm{t})\right)_{\mathrm{t} \geq 0}$ on $\mathrm{E}$. Furthermore, $\mathrm{A}$ has discrete spectrum with eigenvalues $-\mathrm{n}^{2}$, with $\mathrm{n} \in \mathbb{N}$ and corresponding normalized eigenfunctions given by

$$
z_{n}(\xi)=\sqrt{\frac{2}{\pi}} \sin (n \xi)
$$

In addition, $\left\{z_{\mathfrak{n}}: \mathrm{n} \in \mathbb{N}\right\}$ is an orthonormal basis of $\mathrm{E}$. and

$$
\mathrm{T}_{\alpha}(\mathrm{t}) \mathrm{x}=\sum_{\mathrm{n}=1}^{\infty} \exp ^{-\mathrm{n}^{2} \mathrm{t}}\left(x, z_{\mathrm{n}}\right) z_{\mathrm{n}} \text { for } x \in \mathrm{E} \text { and } \mathrm{t} \geq 0 .
$$

Theorem 4.2. Let $\mathcal{B}=\operatorname{BUC}\left(\mathbb{R}_{;} \mathrm{E}\right)$ and $\varphi \in \mathcal{B}$. Assume that condition $\left(\mathrm{H}_{\varphi}\right)$ holds, $\rho_{\mathrm{i}}:[0, \infty) \rightarrow$ $[0, \infty), i=1,2$, are continuous and the functions $a_{i}: \mathbb{R} \rightarrow \mathbb{R}$ are continuous for $i=1,2,3$. Then there exists a unique mild solution of (9). 
Proof. From the assumptions of the above theorem, we have that

$$
\begin{gathered}
f(t, \psi)(\xi)=\int_{-\infty}^{0} a_{1}(s) \psi(s, \xi) d s, \\
g(t, \psi)(\xi)=\int_{-\infty}^{0} a_{3}(s) \psi(s, \xi) d s, \\
\rho(s, \psi)=s-\rho_{1}(s) \rho_{2}\left(\int_{0}^{\pi} a_{2}(\theta)|\psi(0, \xi)|^{2} d \theta\right),
\end{gathered}
$$

are well defined functions, which permit to transform system (9) into the abstract system (1) - (2). Moreover, the function $f$ is bounded linear operator. Now, the existence of a mild solutions can be deduced from a direct application of Theorem 2.12

We have the following result.

Corollary 4.3. Let $\varphi \in \mathcal{B}$ be continuous and bounded. Then the problem (1) - (2) has a unique mild solution on $(-\infty,+\infty)$.

Acknowledgement: The second author would like to express his thanks to Prof. K. Ezzinbi for his invitation and the warm hospitality.

\section{Received: March 2014. Accepted: September 2014.}

\section{References}

[1] S. Abbas, M. Benchohra and G.M. N'Guérékata, Topics in Fractional Differential Equations, Springer, New York, 2012.

[2] M. Adimy and K. Ezzinbi, The basic theory of abstract semilnear functional differntial equations with nondense domain, in "Delay Differential Equations with Applications", ed by O. Arino, M. L. Hbid and E. Ait Dads, NATO Science Series Ш: Mathematics, Physics and Chemistry, Vol.205, (2006), Springer, Berlin, pp.347-407.

[3] M. Adimy, H. Bouzahir and K. Ezzinbi, Local Existence for a Class of Partial Neutral Functional Differential Equations With Infinite Delay, Differ. Equ. Dyn. Syst. 12 (2004), 353-370.

[4] M. Adimy, H. Bouzahir and K.Ezzinbi, Existence and Stability for Some Some Neutral Functional Differential Equations With Infinite Delay, J. Math. Anal. Appl. 294 (2004), 438-461.

[5] R. P. Agarwal, M. Belmekki, M. Benchohra, A survey on semilinear differential equations and inclusions involving Riemann-Liouville fractional derivative, Adv. Difference Equ. 2009, Art. ID $981728,47 \mathrm{pp}$. 
[6] R. P. Agarwal, B. de Andrade, and G. Siracusa. On fractional integro-differential equations with state-dependent, Comput. Math. Appl. 62 (2011), 1143-1149.

[7] K. Aissani and M. Benchohra, Fractional integro-differential equations with state-dependent delay, Adv. Dyn. Syst. Appl. 9 (2014), 17-30.

[8] E. Ait Dads and K. Ezzinbi, Boundedness and almost periodicity for some state-dependent delay differential equations, Electron. J. Differential Equations. 2002,(67), 13pp.

[9] W. Arendt, C. Batty, M. Hieber and F. Neubrander, Vector-Valued Laplace Transforms and Cauchy Problems, Monographs in Mathematics, 96, Birkhauser, Basel, 2001.

[10] S. Baghli, M. Benchohra, J. J. Nieto, Global uniqueness results for partial functional and neutral functional evolution equations with state-dependent delay. J. Adv. Reas. Differ. Equ. 2 (3) (2010), 35-52.

[11] D. Baleanu, K. Diethelm, E. Scalas and J. J. Trujillo, Fractional Calculus Models and Numerical Methods, World Scientific, New York, 2012.

[12] J. Bélair, Population models with state-dependent delays. Lect. Notes Pure Appl. Math., Dekker, New York, 131 (1990) 165-176.

[13] J. Bélair and M.C. Mackey, Consumer memory and price fluctuations on commodity markets: An integrodifferential model. J. Dynam. Differential Equations 1 (1989), 299-325.

[14] M. Benchohra, J. Henderson, S. K. Ntouyas, A. Ouahab; Existence results for functional differential equations of fractional order with infinite delay, J. Math. Anal. Appl. 338 (2008), 1340-1350.

[15] M. A. Darwish and S.K. Ntouyas, Semilinear functional differential equations of fractional order with state dependent delay, Electron. J. Differential Equations 38 2009, 1-10.

[16] J. V. Devi, V. Lakshmikantham, Nonsmooth and fractional differential equations, Nonlinear Anal. 70 (2009), 4151-4157.

[17] J.P.C. dos Santos, M.M. Arjunan, and C. Cuevas, Existence results for fractional neutral integro differential equations with state-dependent delay, Comput. Math. Appl. 62 (2011), 1275-1283.

[18] R.D. Driver, and M.J. Norris, Note on uniqueness for a one-dimensional two- body problem of classical electrodynamics. Ann. Phys. 42 (1967), 347-351.

[19] M. M. El-Borai, Some probability densities and fundamental solutions of fractional evolution equations, Chaos Solitons Fractals 14 (2002), 433-440.

[20] K. J. Engel and R. Nagel, One-Parameter Semigroups for Linear Evolution Equations, Graduate Texts in Mathemaics,194, Springer-Verlag, New York, 2000. 
[21] M. Frigon and A. Granas, Résultat de type Leray-Schauder pour des contractions sur des espaces de Fréchet, Ann. Sci. Math. Québec 22(1998), 161-168.

[22] J. Hale and J. Kato, Phase space for retarded equations with infinite delay, Funkcial. Ekvac. 21 (1978), 11-41.

[23] E. Hernandez, R. Satkhivel and S. Tanaka Aki, Existence results for impulsive evolution differential equations with state-dependent delay, Electron. J. Differential Equations 2008(28),-11.

[24] R. Hilfer, Applications of Fractional Calculus in Physics, World Scientific, Singapore, 2000.

[25] Y. Hino, S. Murakami and T. Naito, Functional Differential Equations with Unbounded Delay. Springer-Verlag, Berlin, 1991.

[26] F. Kapper and W. Schappacher, Some Considerations to the Fundamental Theory of Infinite Delay Equations, J. Differential Equations. 37 (1980), 141-183.

[27] A. A. Kilbas, H. M. Srivastava and J. J. Trujillo, Theory and Applications of Fractional Differential Equations. North-Holland Mathematics Studies, 204. ELsevier Science B.V. Amsterdam, 2006.

[28] V. Lakshmikantham, S. Leela, J. Vasundhara. Theory of Dynamic Systems. Cambridge Academic Publishers, Cambridge, 2009.

[29] C. Lizama, Regularized solutions for abstract Volterra equations, J. Math. Anal. Appl. 243 (2000), 278-292.

[30] K. Li, J. Peng, Fractional resolvents and fractional evolution equations, Appl. Math. Lett. 25 (2012), 808-812.

[31] M.C. Mackey, Commodity price fluctuations: price dependent delays and non- linearities as explanatory factors. J. Econ. Theory 48 (1989), 497-509.

[32] M.C. Mackey, and J. Milton, Feedback delays and the origin of blood cell dynamics, Comm. Theor. Biol. 1 (1990), 299-327.

[33] G. M. Mophou and G. M. N'Guérékata, Existence of mild solutions of some semilinear neutral fractional functional evolution equations with infinite delay, Appl. Math. Comput. 216 (2010), 61-69.

[34] I. Podlubny, Fractional Differential Equations, Academic Press, San Diego, 1999.

[35] J. Prüss, Evolutionnary Integral Equations and Applications, Monographs Math.87, Birkhauser Verlag, 1993.

[36] K. Schumacher, Existence and Continuous Dependence for Fractional Neutral Evolution Equations, Comput. Math. Appl. 59 (2010), 1063-1077. 
[37] V. E. Tarasov, Fractional Dynamics. Applications of Fractional Calculus to Dynamics of Particles, Fields and Media. Springer, Heidelberg, 2010.

[38] K. Velusamy, R. Murugesu, P. Z. Wang, Existence results for neutral functional fraactional differential equations with state dependendent delay, Malaya J. Mat. 11 (2012), 50-61.

[39] K. Yosida, Functional Analysis $6^{\text {th }}$ edn. Springer-Verlag, Berlin, 1980.

[40] Y. Zhou and F. Jiao, Existence and continuous dependece for differential equations with unbounded delay, Math. Appl. 59 (2010), 1063-1077.

[41] Y. Zhou and F. Jiao, Nonlocal Cauchy problem for fractional evolution equations, Nonlinear Anal.: Real World Appl 11 (2010), 4465-4475. 